Among night shifters, those who perceived that they frequently had excess workload were 4.54 (1.03 - 20.00) times more likely to frequently experience physical symptoms.

Conclusion This study has shown that there is an association between specific occupation hazards and shiftwork with adverse physical health among Filipino female factory workers.

\section{P-50 OCCUPATIONAL MOVEMENTS AND POSTURES OF THE NECK AS RISK FACTORS FOR CERVICAL DISC HERNIATION (CDH).}

'Jonathan Petersen, Charlotte Brauer, Lau Thygesen, Esben Meulengracht Flachs, Christina Bach Lund, Jane Froelund Thomsen. 'Bispebjerg Hospital, Denmark

\subsection{6/OEM-2021-EPI.176}

Introduction Some observational and in vivo studies suggest dynamic neck movements and positions as causes of cervical disc disease. However, epidemiological evidence using objective measurements on neck position and movements as exposure is limited.

Objectives We studied the associations between objectively measured occupational neck movements and postures and $\mathrm{CDH}$.

Methods We formed a cohort of 852,625 Danish workers who ever held at least one of 29 jobs (e.g. dentists, hairdressers, childcare, carpenters) from 1981 to 2016. Representative whole work-day inclinometric measurements using triaxial accelerometers measuring neck angular velocity and posture of the neck were used as exposure. Job titles were retrieved from the Danish Occupational Cohort with eXposure data $(\mathrm{DOC} * \mathrm{X})$ database. First diagnosis of $\mathrm{CDH}$ was retrieved from the Danish National Patient Register. The risk of $\mathrm{CDH}$ by quintiles of cumulated exposure was assessed by incidence rate ratios (IRR), adjusted for age, sex, calendar -year, previous lumbar disc herniation and education, using Poisson regression models.

Results We found 14,000 cases of $\mathrm{CDH}$ during 20.2 million person-years of follow-up. Crude analyses showed an increasing IRR of $\mathrm{CDH}$ with increasing angular velocity of the neck. However, in the fully adjusted model increasing levels of neck angular velocity showed a decreasing risk with IRR 0.90 [95\% confidence interval (CI) 0.86-0.95] when the highest quintile (dynamic work) was compared to the lowest (static work). Similar results were found for extension and flexion of the neck. Multiple sensitivity analyses did not change the results.

Conclusion In this large register-based study, we found no evidence of an increased risk of $\mathrm{CDH}$ in jobs with high velocity of movements or extreme positions of the neck. Other factors than dynamic neck movements and bent neck position seem to be important in the development of $\mathrm{CDH}$.

\section{P-51 REPETITIVE AND FORCEFUL MOVEMENTS OF THE HAND AS PREDICTORS FOR PAIN IN THE DISTAL UPPER EXTREMITIES}

'Jonathan Petersen, Charlotte Brauer, Lau Thygesen, Esben Meulengracht Flachs, Christina Bach Lund, Jane Froelund Thomsen. 'Bispebjerg Hospital, Denmark

\subsection{6/OEM-2021-EPI.177}

Introduction Some evidence exits of an association between measures of occupational use of hand force and repetition and tendinitis of the wrist and epicondylitis of the elbow. However, these studies have often been limited by bias because of self-reported exposure and an outcome sensitive to time fluctuations.

Objectives To investigate occupational repetitive movements and the use of hand force as causes of distal upper extremities musculoskeletal disorders (D-UEMSD).

Methods A cohort of 202,735 workers in a private pension health scheme from 2005 to 2017 in one of 17 jobs (e.g. office work, carpentry, cleaning) was formed. Representative electro-goniometric measurements of wrist angular velocity as a measure for repetition and expert-rated use of hand force were used as exposures. Job titles were retrieved from the Danish Occupational Cohort with eXposure data (DOC*X) database. Outcome was first treatment for D-UEMSD. In a Poisson regression model, the incidence rate ratios (IRRs) of D-UEMSD were adjusted for age, calendar-year, diagnosis of rheumatoid arthritis and arm fractures. In further analyses, wrist velocity or hand force was added.

Results In men, wrist velocity had an IRR of 1.48 (95\%CI 1.15-1.91) when the highest exposure level was compared to the lowest but with no clear exposure-response pattern. The effect became insignificant when adjusted for hand force. Hand force had an IRR of 2.65 (95\%CI 2.13-3.29) for the highest vs. the lowest exposure with an exposure-response pattern, which remained after adjustment for wrist velocity. Among women, no increased risk was found for hand force, while wrist velocity showed a significantly protective association with D-UEMSD.

Conclusions In men, occupational exposure to hand force more than doubled the risk of seeking treatment for DUEMSD. The results for exposure to repetition were less clear. In women, we could not find any indications of an increased risk neither for force nor repetition.

\section{P-52 PROSPECTIVE ASSOCIATIONS OF MULTIPLE EXPOSURES TO PSYCHOSOCIAL WORK FACTORS WITH WELL-BEING AMONG EMPLOYEES IN FRANCE}

'Sandrine Bertrais, Nora Hérault, Jean-François Chastang, Isabelle Niedhammer. 'Inserm DR GRAND OUEST, France

\subsection{6/OEM-2021-EPI.178}

Objective Literature reviews showed adverse effects of high job demands, low job control, and low social support at work on well-being in working populations. Other psychosocial work factors (PWFs) remain understudied in association with well-being. We aimed to examine the prospective associations of a large variety of PWFs and other occupational exposures with poor well-being, as evaluated by the WHO-5 well-being index. An additional objective was to explore the effects of multiple occupational exposures on this outcome.

Methods The study sample consisted of 15776 employees aged 15-65 years (9181 women, 8579 men) included in the representative sample of the French national survey on working conditions and followed up from 2013 to 2016. Participants were classified as having poor well-being if they had a WHO-5 score below 13. Occupational exposures included 20 PWFs which were studied separately and then grouped into five dimensions (work demands, work organization and job content, interpersonal relations and leadership, work-individual interface, workplace violence), 4 factors related to working 